\title{
Output Polarization Control of Fiber DFB Laser Using Injection Locking
}

\author{
W. H. Chung, L. Y. Chan, H. Y. Tam, Senior Member, IEEE, P. K. A. Wai, Senior Member, IEEE, and \\ M. S. Demokan, Senior Member, IEEE
}

\begin{abstract}
Experimental results of a distributed feedback fiber laser injection locked to a Fabry-Pérot laser diode are presented. By adjusting the injection power of the fiber laser to the Fabry-Pérot laser, high quality laser output with a degree of polarization larger than $95 \%$ and sidemode suppression ratio higher than $45 \mathrm{~dB}$ was achieved. A 3.8-dB power penalty improvement was also demonstrated in a $41-\mathrm{km} 10-\mathrm{Gb} / \mathrm{s}$ transmission experiment.
\end{abstract}

Index Terms-Fabry-Pérot laser, fiber DFB laser, injection locking.

\section{INTRODUCTION}

D ISTRIBUTED feedback fiber lasers (DFB-FLs) based on fiber Bragg gratings written in $\mathrm{Er}^{3+}$ - or $\mathrm{Er}^{3+}-\mathrm{Yb}^{3+}$-doped fibers are promising candidates for optical communication and sensor applications because of their single longitudinal mode output, narrow linewidth, and ease of fabrication with highly accurate lasing wavelength obtained by current phase mask writing method. Most fiber lasers' output consists of two orthogonal linear polarizations if they were fabricated using low birefringent fiber. These two polarization modes are sensitive to external perturbations which make them suitable for strain [1], [2], differential pressure, and force [3], as well as simultaneous strain and temperature measurement [4]. In contrast, single polarization operation is more desirable for optical communication applications.

Several techniques have been developed to make fiber lasers operate in a single polarization. They include twisting the fiber lasers [5], introducing a birefringence phase shift in the fiber lasers [6], and self-injection locking [7], [8] that employ a feedback to the cavity which can be either polarization dependent or independent. In this letter, we investigate the use of an active injection-locking technique. By injecting the output of a DFB-FL to a Fabry-Pérot laser diode (FP-LD), we show that stable and highly polarized output can be achieved. A 3.8-dB power penalty improvement was also demonstrated in a $41-\mathrm{km}$ $10-\mathrm{Gb} / \mathrm{s}$ transmission test.

\footnotetext{
Manuscript received December 3, 2001; revised March 4, 2002. This work was supported by the Research Grant Council of the Hong Kong Special Administrative Region, China (Project PolyU5132/99E).

W. H. Chung, H. Y. Tam, and M. S. Demokan are with the Photonics Research Center, Department of Electrical Engineering, The Hong Kong Polytechnic University, Hong Kong, China (e-mail: eewhchun @inet.polyu.edu.hk).

L. Y. Chan and P. K. A. Wai are with the Photonics Research Center, Department of Electronic and Information Engineering, The Hong Kong Polytechnic University, Hong Kong, China.

Publisher Item Identifier S 1041-1135(02)04609-8.
}

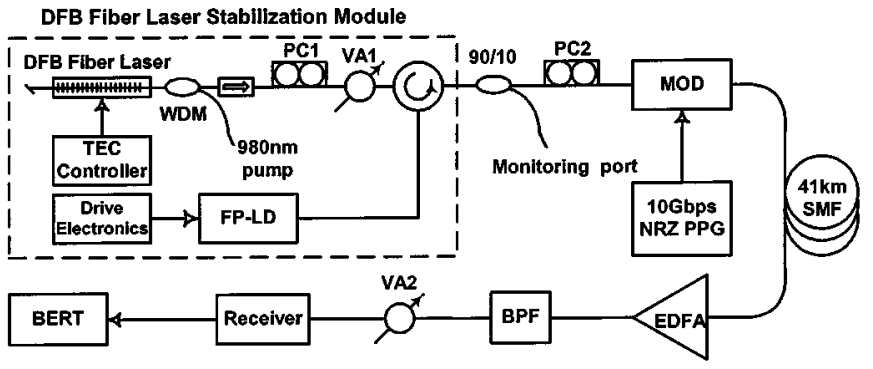

Fig. 1. Experimental setup. WDM: Wavelength-division multiplexer. PC: Polarization controller. VA: Variable attenuator. 90/10: 90/10 coupler. MOD: Modulator. BPF: Bandpass filter.

\section{EXPERIMENTS AND RESULTS}

Fig. 1 shows the experimental configuration used to demonstrate the stabilization of the polarization of a DFB-FL output. The DFB-FL was fabricated by scanning a phase mask in a $5-\mathrm{cm}$ length hydrogenated $\mathrm{Er}-\mathrm{Yb}$ fiber by a $248-\mathrm{nm}$ excimer laser. We then introduced an additional $\pi$ phase shift in the fiber at $28 \mathrm{~mm}$ from the output end of the laser by shifting the phase mask half a grating pitch using a high precision piezoelectric stage during the UV scanning process. An isolator was inserted after the wavelength-division multiplexer, used for pumping the fiber laser and the unused end of the DFB-FL was terminated by an index matching gel to prevent any unwanted feedback that may destabilize the fiber laser. We epoxied the DFB-FL on an aluminum plate which is temperature stabilized to $25 \pm 0.1^{\circ} \mathrm{C}$ in order to avoid the pump induced frequency instability effect [9]. The measured lasing wavelength and linewidth of the DFB-FL were $1551.5 \mathrm{~nm}$ and less than $500 \mathrm{kHz}$, respectively. The measured linewidth was limited by the spectral linewidth of the tunable diode laser used for heterodyne beating. Fig. 2(a) shows the output spectrum of the DFB-FL. The output power and sidemode suppression ratios (SMSR) of the DFB-FL were measured to be $5 \mathrm{~mW}$ and $75 \mathrm{~dB}$, respectively. The pump power of the 980-nm pump laser was $70 \mathrm{~mW}$. Dual polarization operation was confirmed by heterodyning with a single-mode external cavity tunable diode laser using a fast detector. We observed a frequency difference of $850 \mathrm{MHz}$ between the two polarization modes as shown in Fig. 3(a), which implies the effective birefringence of the DFB-FL is $6.37 \times 10^{-6}$.

We then injected the output power of the DFB-FL to the FP-LD employing a polarization independent circulator. We monitored the injection locked signal from the output port of the circulator. The FP-LD was a double channel planar buried 

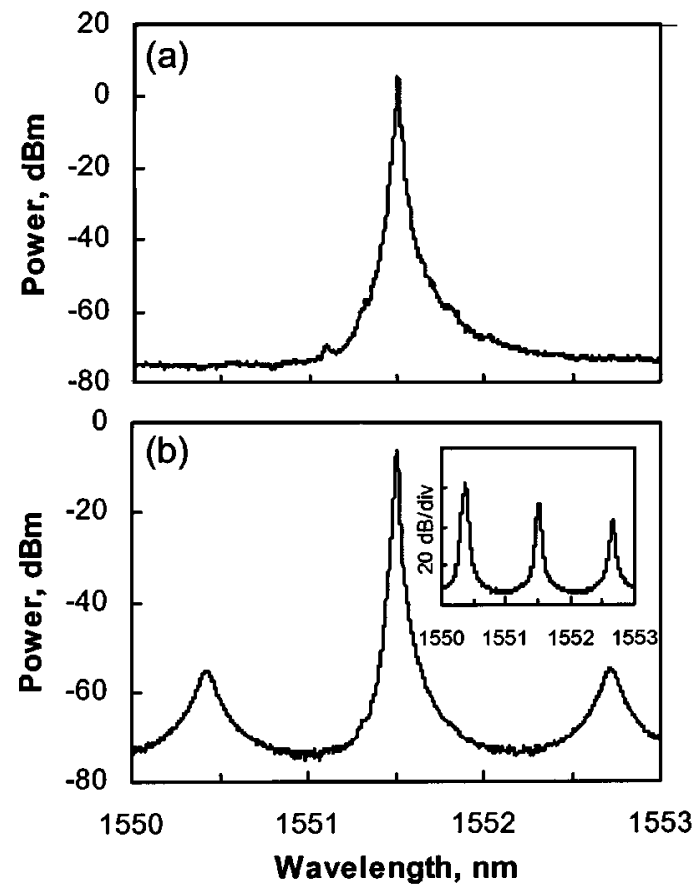

Fig. 2. Optical spectra of (a) DFB-FL, (b) injection locked FP-LD. Inset: Spectra of the FP-LD not injection locked.
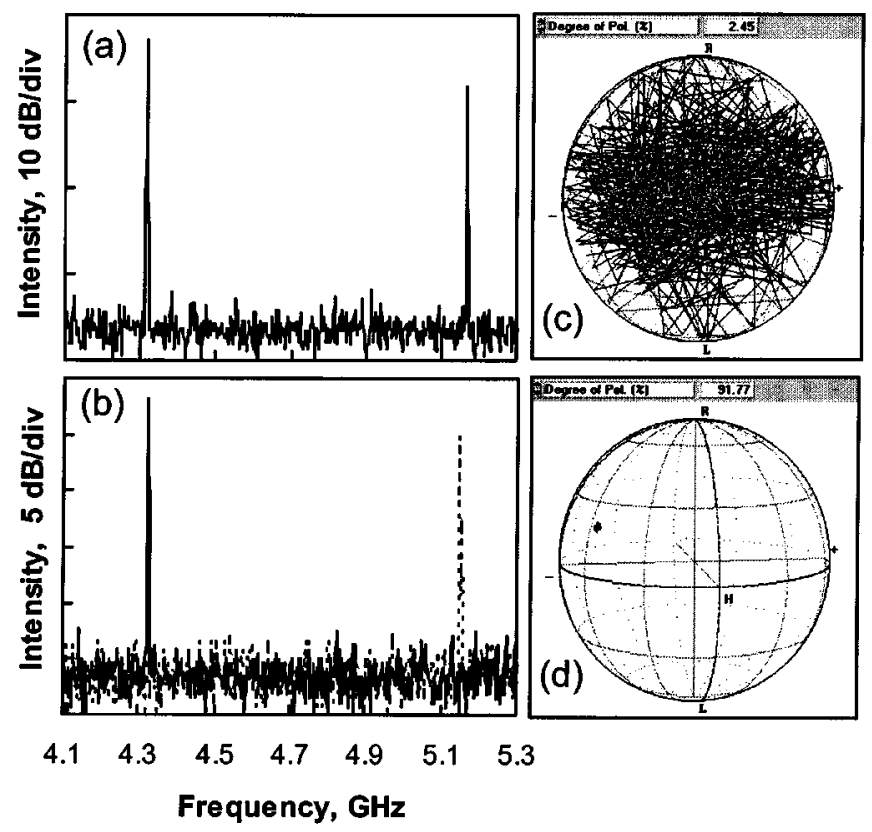

Fig. 3. RF beating spectra of the DFB-FL. (a) Without injection locking, showing dual polarization output. (b) With injection locking, showing single output polarization output (solid or dashed trace). The measured Poincaré spheres for the DFB-FL output showing (c) fluctuation in the state of polarizations without FP-LD and (d) stable state of polarizations with FP-LD.

heterostructure (DC-PBH) type diode laser with center wavelength of $1548.7 \mathrm{~nm}$ and a threshold current $\left(I_{\mathrm{th}}\right)$ of $11 \mathrm{~mA}$. Fig. 2(b) shows the spectrum at the monitoring port which demonstrates the effect of injection locking. The inset is the original FP-LD spectrum. Injection locking was achieved by fine-tuning the spectrum of the FP-LD by varying temperature. When injection locking was achieved, the mode located at

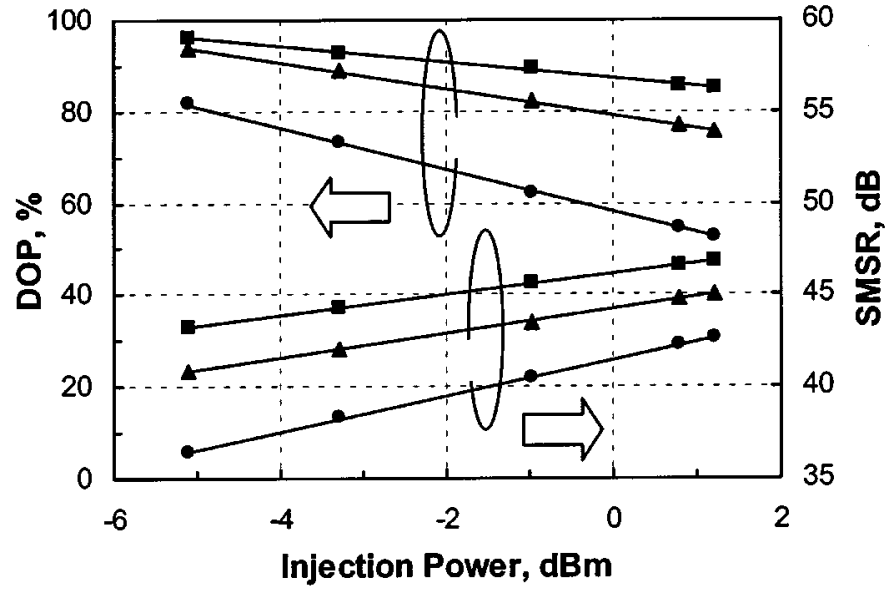

Fig. 4. Degree of polarization (DOP) and side mode suppression ratio (SMSR) as a function of injection power (output power of the DFB-FL) with different FP-LD bias current $\left(\boldsymbol{\square} 1.8 I_{\mathrm{tl}_{1}} ; \boldsymbol{\Delta} 1.45 I_{\mathrm{tl}_{1}} ; \bullet 1.1 I_{\mathrm{tl}_{1}}\right)$.

$1551.5 \mathrm{~nm}$ was amplified by $25 \mathrm{~dB}$. All sidemodes are strongly suppressed by $20 \mathrm{~dB}$ and they are also red shifted by $0.07 \mathrm{~nm}$ [10]. The SMSR of the injection-locked output was $45 \mathrm{~dB}$, which can be improved to about $65 \mathrm{~dB}$ by filtering the output with a thin-film bandpass filter. Note that the linewidth of the injection locked output was narrower as compared to the linewidth of the original FP-LD. Heterodyning the injection locked laser with an external cavity tunable diode laser showed that either one of the polarization modes [solid and dashed lines in Fig. 3(b)] of the DFB-FL can be selected to operate by adjusting the polarization controller (PC1).

Polarization characteristics of the original DFB-FL and the injection-locked laser were measured by a polarization analyzer (Profile PAT 9000B). Fig. 3(c) shows the state of polarization (SOP) of the original DFB-FL on a Poincaré sphere. The measurement period was $30 \mathrm{~s}$ and the sampling rate was $30 \mathrm{~Hz}$. The degree of polarization (DOP) was measured to be $2.45 \%$ and the SOP was unstable throughout the measuring duration. On the other hand, the SOP of the injection locked laser was very stable over the same time interval and the DOP was $91.77 \%$ as shown in Fig. 3(d). This can be explained by considering that the FP-LD is an internally strained semiconductor laser operating in single transverse electric (TE) polarization [11], and when one of the polarization modes of the DFB-FL is aligned to the TE mode of the FP-LD, it will be strengthened resonantly by the injection locking effect whereas the other orthogonally aligned polarized mode will be strongly diminished.

To study the injection locking phenomenon, different bias currents were applied to the FP-LD and the output power of the DFB-FL was varied. Fig. 4 depicts the measured DOP and SMSR as a function of the injection power by adjusting the variable attenuator (VA1) which changes the power output from the DFB-FL with three different bias currents $\left(1.1 I_{\mathrm{th}}, 1.45 I_{\mathrm{th}}\right.$ and $1.8 I_{\text {th }}$ ) of the FP-LD. We found that the sidemodes power decreases (i.e., increase in SMSR) when the injection power or the FP-LD bias current increases. SMSR of $47 \mathrm{~dB}$ was obtained with the injection power of $1.2 \mathrm{dBm}$ and FP-LD biased at $1.8 I_{\mathrm{th}}$. The DOP, on the other hand, increases when FP-LD bias current increases but decreases when injection power increases. This 


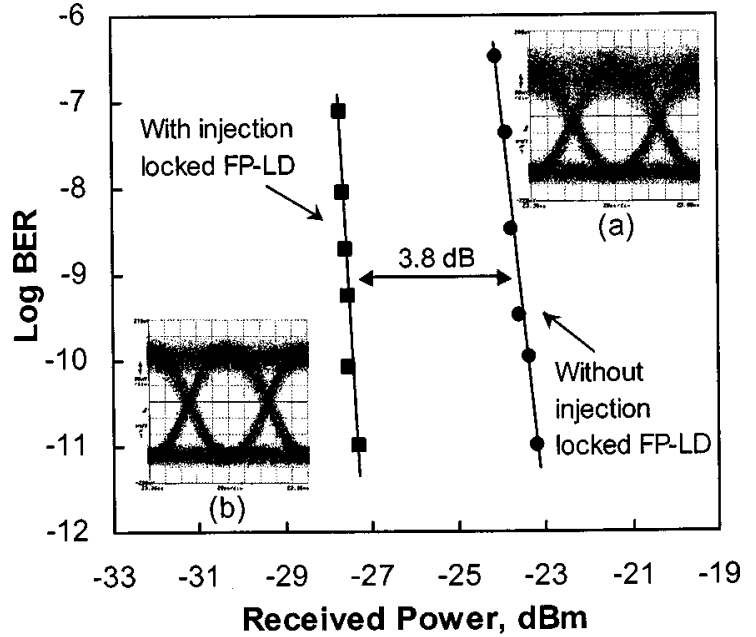

Fig. 5. BERs and eye diagrams of the DFB-FL (a) without and (b) with injection locking.

can be explained by considering that due to the geometrical structure of the FP-LD, its output is single polarization mode and when the effect of injection locking reduces via the decrease in power of the DFB-FL, the output of the circulator becomes dominantly a single polarization mode. Therefore, optimization between the bias current of the FP-LD and the injection power of the DFB-FL are required in order to optimize the DOP and SMSR of the injection-locked laser. We note that the injection bandwidth of the FP-LD is about $30 \mathrm{GHz}$ [10] which is significantly higher than the $10 \mathrm{MHz}$ reported in [7] for the injection locking of a fiber DFB laser. The FP-LD in this experiment acts as a polarization filter as well as an amplifying medium for the DFB-FL when it is injection locked. Thus, the polarizer used in the self-injection locking technique of [7] is eliminated in our experiment at the expense of higher insertion loss of the circulator used for injection locking.

The transmission performance of using only the DFB-FL and then the injection locked FP-LD/DFB-FL as sources were compared by the experiment setup shown in Fig. 1. The injection power of the DFB-FL was set to $-5 \mathrm{dBm}$ and the bias current of the FP-LD was $20 \mathrm{~mA}\left(1.8 I_{\mathrm{th}}\right)$, the corresponding DOP and SMSR of the injection locked laser output were $97 \%$ and $43 \mathrm{~dB}$. Insets of Fig. 5 shows the 10-Gb/s NRZ externally modulated eye diagrams (a) when only the DFB-FL is used, and (b) the injection locked laser is used recorded by a sampling oscilloscope operated in 10-s persistence mode (upper right and lower left, respectively). Obviously, dual polarization output of the DFB-FL causes amplitude jitter after the polarization-sensitive modulator and leads to partial eye closure. An open eye was obtained from the injection locked laser for its highly polarized output. The lasers were then modulated by $2^{31}-1$ pseudorandom bit sequence and bit error rates (BERs) were recorded after transmitting through $41-\mathrm{km}$ standard single-mode fiber. Fig. 5 shows the BERs of the two different configurations. The 3.8-dB power penalty improvement was achieved with BER of $10^{-9}$ when the injection locked output was transmitted.

\section{CONCLUSION}

We presented an alternative method to modify the output polarization of a DFB-FL. An active injection-locking technique was employed. By adjusting the operating current of the FP-LD and the output power of the DFB-FL, we achieved nearly linear polarized laser output with DOP larger than $95 \%$ and SMSR higher than $45 \mathrm{~dB}$. The performance of the resulting source using this technique is, therefore, comparable with that of semiconductor DFB laser diodes used for optical communication purposes. We have also shown that this technique can eliminate the amplitude jitter of an externally modulated DFB-FL, and observed a $3.8-\mathrm{dB}$ power penalty improvement in a $10-\mathrm{Gb} / \mathrm{s}$ 41-km transmission experiment. Our results indicate that a highly polarized multiwavelength source can be realized by injection locking of a single low-cost FP-LD with several fiber lasers, which will find potential application in dense WDM system, as well as component characterization.

\section{REFERENCES}

[1] G. A. Ball, G. Meltz, and W. W. Morey, "Polarimetric heterodyning Bragg-grating fiber-laser sensor," Opt. Lett., vol. 18, pp. 1976-1978, 1993.

[2] H. K. Kim, S. K. Kim, H. G. Park, and B. Y. Kim, "Polarimetric fiber laser sensors," Opt. Lett., vol. 18, pp. 1465-1467, 1993.

[3] J. L. Kringlebotn, W. H. Loh, and R. I. Laming, "Polarimetric $\mathrm{Er}^{3+}$-doped fiber distributed-feedback laser sensors for differential pressure and force measurements," Opt. Lett., vol. 21, p. 1869, 1996.

[4] O. Hadeler, E. Rønnekeiv, M. Ibsen, and R. I. Laming, "Polarimetric distributed feedback fiber laser sensor for simultaneous strain and temperature measurements," Appl. Opt., vol. 38, pp. 1953-1957, 1999.

[5] Z. E. Harutjunian, W. H. Loh, R. I. Laming, and D. N. Payne, "Single polarization twisted distributed feedback fiber laser," Electron. Lett., vol. 32, pp. 346-348, 1996.

[6] H. Storøy, B. Sahlgren, and R. Stubbe, "Single polarization fiber DFB laser," Electron. Lett., vol. 33, pp. 56-58, 1997.

[7] S. Yamashita and G. J. Cowle, "Single-polarization operation of fiber distributed feedback (DFB) lasers by injection locking," J. Lightwave Technol., vol. 17, pp. 509-513, Mar. 1999.

[8] K. Hsu and S. Yamashita, "Single-polarization generation in fiber Fabry-Perot laser by self-injection locking in short feedback cavity," $J$. Lightwave Technol., vol. 19, pp. 520-526, Apr. 2001.

[9] W. S. Man, Y. Z. Xu, H. Y. Tam, and M. S. Demokan, "Frequency instability in $\mathrm{Er} / \mathrm{Yb}$ fiber grating lasers due to heating by nonradiative transitions," IEEE Photon. Techol. Lett., vol. 11, pp. 1390-1392, Nov. 1999.

[10] C. H. Henry, N. A. Olsson, and N. K. Dutta, "Locking range and stability of injection locked $1.54 \mu \mathrm{m}$ InGaAsP semiconductor lasers," IEEE J. Quantum Electron., vol. QE21, pp. 1152-1156, Aug. 1985.

[11] B. M. Yu and J. M. Liu, "Polarization-dependent gain, gain nonlinearities, and emssion characteristics of internally strained InGaAsP/InP semiconductor lasers," J. Appl. Phys., vol. 69, pp. 7444-7459, 1991. 